\title{
Vibrational density of states of the hydrogen sites in hydrogen-bonded molecular solids*
}

\author{
C. Andreani ${ }^{\mathrm{a}}$, V. Merlo ${ }^{\mathrm{a}}$, P. Postorino ${ }^{\mathrm{b}}$ and M.A. Ricci ${ }^{\mathrm{b}}$ \\ a Dipartimento di Fisica, Università degli Studi di Tor Vergata, Rome (Italy) \\ 'Dipartimento di Fisica, Universitò degli Studi di L'Aquila, L'Aquila (Italy) \\ (Received 9 November 1990)
}

\begin{abstract}
Inelastic neutron scattering measurements of the incoherent dynamical structure factor of hydrogen-bonded molecular solids are reviewed. Particular attention is devoted to ice and ice-like polycrystalline samples. Their vibrational density of states is discussed in terms of the influence of proton disorder, anharmonicity and fluctuations of the force constants on the width and shape of the band.
\end{abstract}

\section{INTRODUCTION}

Since 1980, with the advent of intense beams of epithermal neutrons from pulsed spallation sources in the USA, Europe and Japan [1], it has become possible to measure the inelastic neutron scattering from molecular solids up to the region of the intramolecular vibrational frequencies. The measured spectrum can be directly related to some projected vibrational density of states in the case of purely incoherent scatterers. This is possible because the response of the sample is not weighted by its frequency-dependent properties, as in the case of Raman scattering.

Among molecular solids the hydrogenated ones are the most promising owing to the high incoherent cross-section of hydrogen. In this paper inelastic neutron scattering measurements on polycrystalline samples of $\mathrm{H}_{2} \mathrm{O}$ [2], $\mathrm{D}_{2} \mathrm{O}$ [3], $\mathrm{KH}_{2} \mathrm{PO}_{4}$ [4] and $\mathrm{H}_{2} \mathrm{SO}_{4}$ [5] are reviewed. We will focus essentially on the $\mathrm{X}-\mathrm{H}$ vibrational spectrum since, in principle, this can be strongly affected by both proton disorder and hydrogen motion anharmonicity.

*Presented at the International Symposium on Hydrogen Bond Physics held at Il Ciocco, Barga, Italy, 11-14 September 1990. 
DERIVATION OF THE VIBRATIONAL DENSITY OF STATES $g(\omega)$ : BASIC THEORY

For the case of hydrogenous materials the incoherent double-differential cross-section, $\mathrm{d}^{2} \sigma / \mathrm{d} \Omega \mathrm{d} \omega$, can be expressed in terms of the hydrogen-sites correlation functions [6]

$$
\begin{aligned}
\left(\frac{\mathrm{d}^{2} \sigma}{\mathrm{d} \Omega \mathrm{d} \omega}\right)_{\text {incoh }}^{\text {inel }}= & \frac{\sigma_{\mathrm{H}}^{i} k_{1}}{4 \pi k_{0}} \int_{-\infty}^{\infty} \mathrm{d} t \frac{1}{2 \pi \hbar} \exp (-\mathrm{i} \omega t) \exp [-2 W(\vec{Q})] \\
& \times \sum_{j}\left[\exp <\left(\vec{u}_{j}(0) \times \vec{Q}\right)\left(\vec{u}_{j}(t) \times \vec{Q}\right)>-1\right]
\end{aligned}
$$

where $k_{0}$ and $k_{1}$ are the incident and scattered neutron wavevectors, $\sigma_{\mathrm{H}}^{i}$ is the incoherent scattering cross-section for hydrogen, $u_{j}$ are the displacements of the jth hydrogen atom and $W(\bar{Q})$ is the Debye-Waller factor. Equation $(1)$ is exact within the harmonic approximation for incoherent scattering.

If we now expand the exponential in eqn. (1), retaining only the one-phonon term, it is possible to express the double-differential cross-section in terms of the one-phonon incoherent scattering function, $S_{\text {incoh }}^{+1}(Q, \omega)$

$\left(\frac{\mathrm{d}^{2} \sigma}{\mathrm{d} \Omega \mathrm{d} \omega}\right)_{\text {incoh }}^{\text {inel }}=\frac{\sigma_{\mathrm{H}}^{i} k_{1}}{4 \pi k_{0}} S_{\text {incoh }}^{+1}(Q, \omega)$

The spectrum which one measures experimentally, $S_{\text {exp }}(Q, \omega)$, will coincide with the right-hand side of eqn. (2) if the following conditions hold: (i) the sample is a purely incoherent scatterer or the scattering from the partially coherent sample can be treated within the incoherent approximation; (ii) the multiple scattering events are negligible; and (iii) the anharmonic effects are negligible.

As far as item (i) is concerned, this is indeed the case for samples containing hydrogen; while in the case of deuterated species it can be shown [3] that the incoherent approximation holds in the $\mathrm{X}-\mathrm{D}$ stretching frequency region. The multiple scattering contribution to the experimental spectrum (item (ii)) is in fact a large fraction of the total intensity in the case of hydrogenous samples: it can be of the order of $20 \%$ [2] even for samples a few millimeters thick. However, a procedure to account for this unwanted contribution has been successfully applied to the whole series of data considered here. The multiple scattering, $S_{\exp }^{\text {mult }}(Q, \omega)$, has been estimated in the hypothesis to produce a contribution which is almost isotropic and essentially due to two scattering events $90^{\circ}$ apart. These conditions hold very well in the four experiments described here (direct scattering geometry and light mass scatterers). Details of the multiple scattering correction procedure are reported in refs. 7 and 8.

Once the multiple scattering contribution is subtracted from the experimental $S_{\text {exp }}(Q, \omega)$ the single scattering $S_{\exp }^{\text {single }}(Q, \omega)$, which should now coincide with the right-hand side of eqn. (2), can be related to the density of states $g(\omega)[5]$ via 
$\lim _{Q \rightarrow 0} \frac{S_{\text {exp }}^{\text {single }}(Q, \omega)}{Q^{2}} 2 M \omega \frac{\exp [2 W(Q)]}{n(\omega)+1}=g(\omega)$

where $n(\omega)$ is the Bose population factor and $M$ is the mass of the hydrogen. Equation (3) is used to evaluate the $g(\omega)$ by performing the extrapolation to zero $Q$-values. In this procedure a possible error can arise if the available $Q$-range is not sufficiently close to zero and a large contribution from multiphonon terms is present. In order to minimize the latter effect all experiments were performed at low temperatures. In particular, as far as the intramolecular frequency region is concerned, one has to check that the intensity follows the $Q^{2}$ behavior in the available experimental $Q$-range. If this is the case, then the small multiphonon component present in the spectrum can be readily eliminated in the $Q \rightarrow 0$ extrapolation procedure.

As far as item (iii) is concerned, all the data presented here have been analyzed in the harmonic approximation. An internal consistency check of this hypothesis can be performed if one uses the expressions which relate $S_{\text {incoh }}^{+1}(Q, \omega)$ to the $g(\omega)$ extracted from eqn. $(3)$ :

$$
\begin{aligned}
S_{\text {incoh }}^{+1}(Q, \omega)= & \int_{-\infty}^{\infty} \mathrm{d} t \frac{1}{2 \pi \hbar} \exp (-\mathrm{i} \omega t) \exp [-2 W(\vec{Q})] \\
& \times \sum_{j}\left[\exp <\left(\vec{u}_{j}(0) \times \vec{Q}\right)\left(\vec{u}_{j}(t) \times \vec{Q}\right)>\right]
\end{aligned}
$$

and

$$
<\left(\vec{u}_{j}(0) \times \vec{Q}\right)\left(\vec{u}_{j}(t) \times \vec{Q}\right)>=\frac{\hbar Q^{2}}{2 M} \int_{-\infty}^{\infty} \mathrm{d} \omega \frac{g(\omega)}{\omega} n(\omega) \exp (-i \omega t)
$$

The $S_{\text {incoh }}^{+1}(Q, \omega)$ derived following eqns. (4a) and (4b) can then be compared with the $S_{\text {exp }}^{\text {single }}(Q, \omega)$ in order to quantitatively assess the anharmonicity contribution in the measured spectrum.

\section{EXPERIMENTAL SET-UP}

The possibility of deducing a reliable density of states from neutron scattering experiments is strongly connected to the availability of the scattering function at low momentum transfer, $Q$. The neutron spectrometers which accomplish this requirement are the direct geometry machines. In these spectrometers a phased Fermi chopper produces pulses of monochromatic neutrons incident on the sample [9]. The energy and momentum transfer of the scattered neutrons are determined by time-of-flight techniques in an assembly of detectors (Fig. 1). In this scattering geometry, if one uses a high incident energy in conjunction with low scattering angles, it is possible to measure the vibrational spectrum of a polycrystalline solid at relatively low $Q$ [10]. Examples of this 


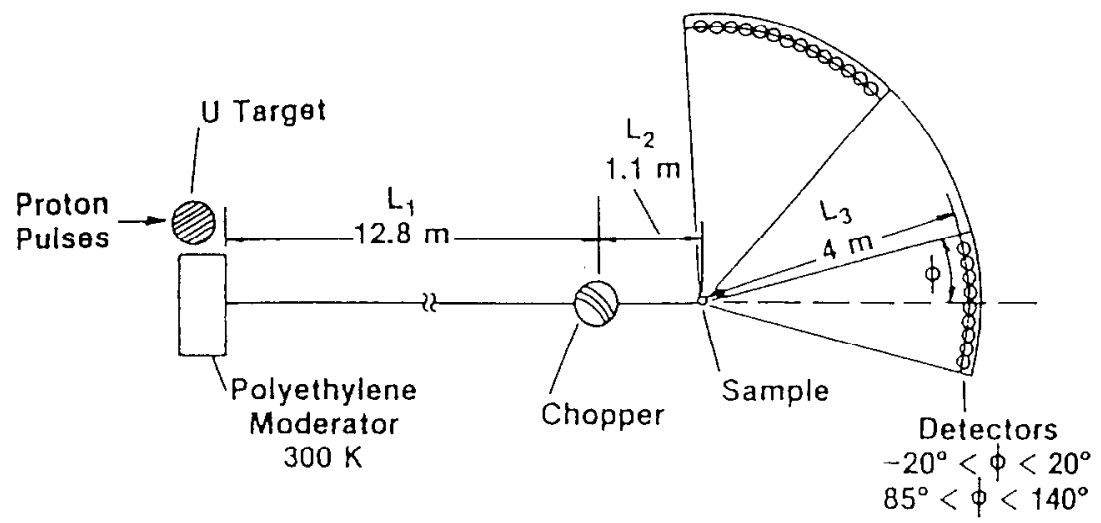

Fig. 1. A schematic diagram of the High Resolution Medium Energy Chopper Spectrometer (HRMECS) installed at IPNS (Argonne National Laboratory).

kind of spectrometers are HRMECS and HET installed at IPNS (Argonne, USA) and ISIS (Rutherford Appleton Laboratory, UK) respectively.

Another requirement, essential for hydrogenous samples, is the employment of a flat geometry assembly. This gives the possibility of using samples of limited thickness yet covering most of the incident beam area (typically $40 \times 40$ $\mathrm{mm}^{2}$ ), thus allowing a higher count rate and analytic experimental corrections (absorption, self shielding, etc.). Furthermore, in this sample geometry a straightforward experimental evaluation of the multiple scattering contribution is possible, from the spectrum measured at $2 \theta=90^{\circ}[7,8]$.

\section{EXPERIMENTAL RESULTS AND DISCUSSION}

The first experiment we want to discuss was performed on polycrystalline $\mathrm{H}_{2} \mathrm{O}$ where the hydrogen atoms are in a disordered arrangement. The threedimensional plot of the experimental data is shown in Fig. 2. The translational band at $100 \mathrm{meV}$, the librational band at $150 \mathrm{meV}$, the bending at $220 \mathrm{meV}$ and the $\mathrm{O}-\mathrm{H}$ stretching at $417 \mathrm{meV}$ are clearly visible. We can also observe that in the stretching region the intensity at fixed energy varies with $Q$ less than it does in the translational energy region. This indicates that, apart from the last band, the multiphonon component is small and this allows us to treat the data in terms of eqn. (3). In Fig. 3 the $\mathrm{H}_{2} \mathrm{O}$ density of states, $g(\omega)$, is shown for the librational and vibrational energy regions.

The $\mathrm{O}-\mathrm{H}$ stretching appears as an unstructured band with a breadth of about $45 \mathrm{meV}$. A possible contribution to the intrinsic width of the stretching band can derive from the presence of disorder and proton motion anharmonicity. In order to check these points an expèriment on polycrystalline $\mathrm{H}_{2} \mathrm{SO}_{4}$ [5], which shows an ordered arrangement of hydrogens, has been performed. In Fig. 4 the 


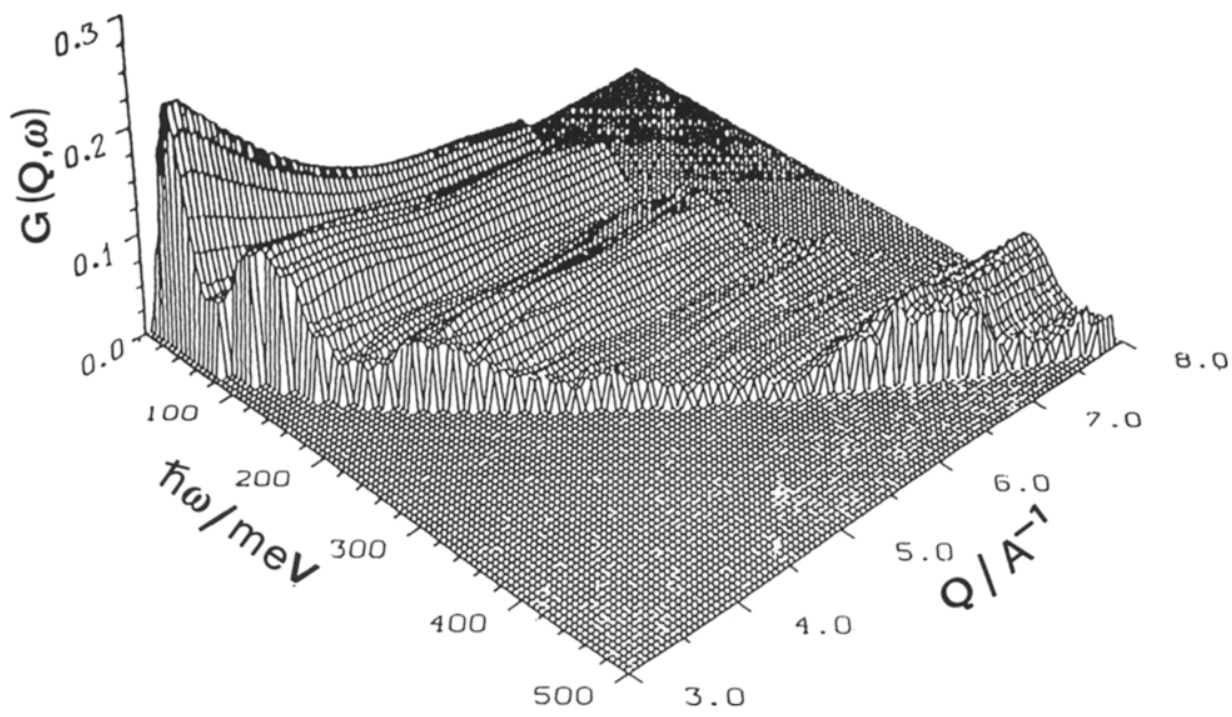

Fig. 2. Neutron weighted function $G(Q, \omega)=\omega^{2} / Q^{2} S_{\text {exp }}(Q, \omega)$.

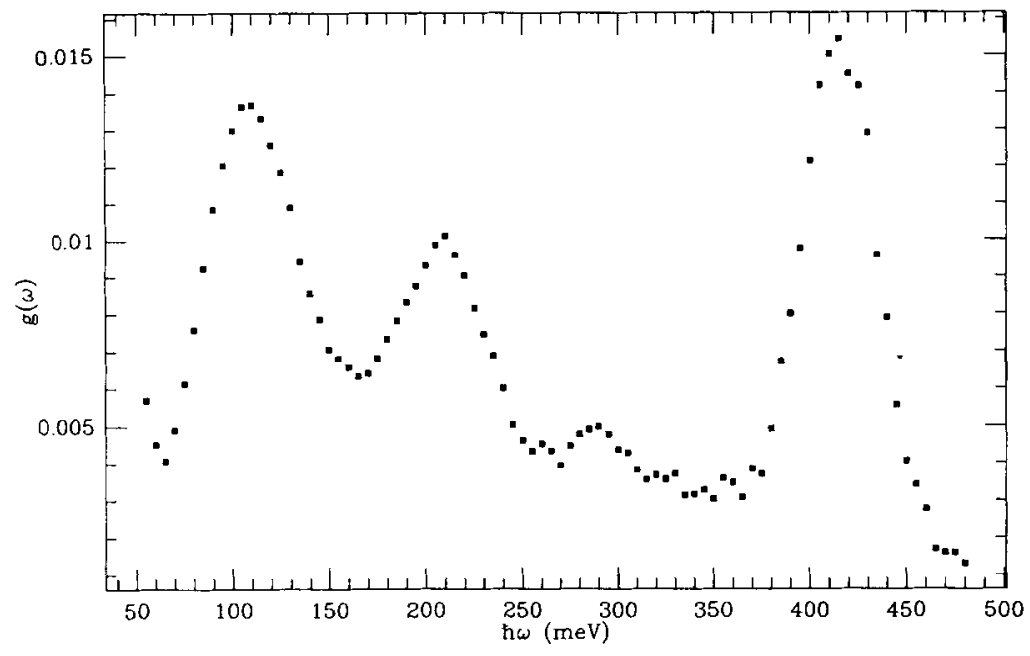

Fig. 3. The hydrogen projected density of states, $g(\omega)$, for ice $I_{h}[2]$.

$g(\omega)$ is plotted for two different incident energy values. No evident sharpening of the stretching band is observed, despite the ordered hydrogen lattice. Furthermore a check for a possible anharmonic contribution to the intrinsic breadth of the $g(\omega)$ has been performed in this case (according to eqns. (4a) and (4b)) and the results are shown in Fig. 5. The intramolecular modes $(\hbar \omega=150$ and $\hbar \omega=375 \mathrm{meV}$ ) are well reproduced within the harmonic model, whereas there 


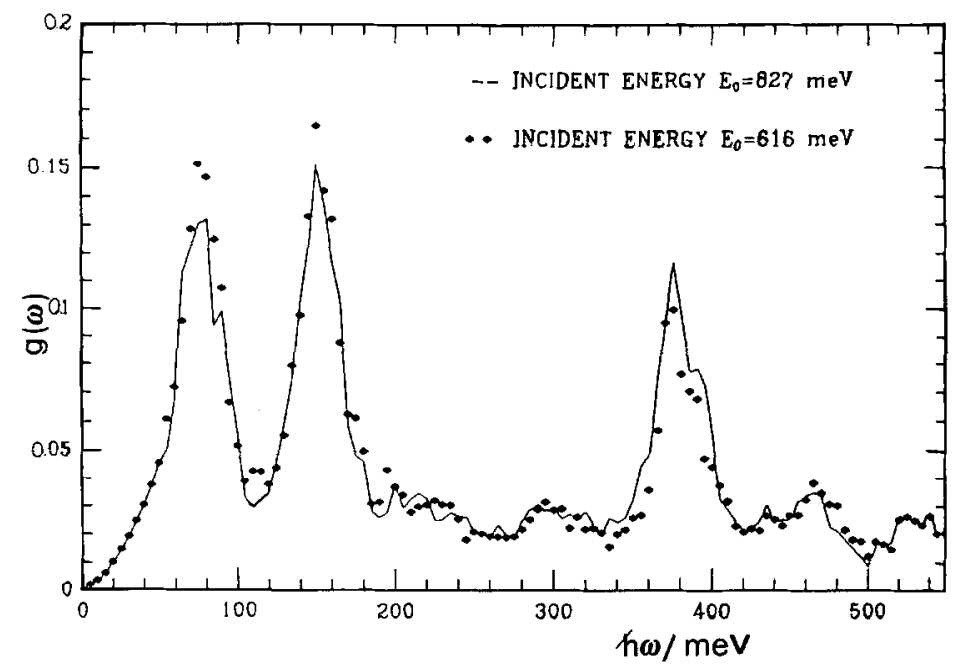

Fig. 4. The hydrogen projected density of states, $g(\omega)$, for polycrystalline $\mathrm{H}_{2} \mathrm{SO}_{4}$ at $T=20 \mathrm{~K}$ for two different values of the incident energies [5].

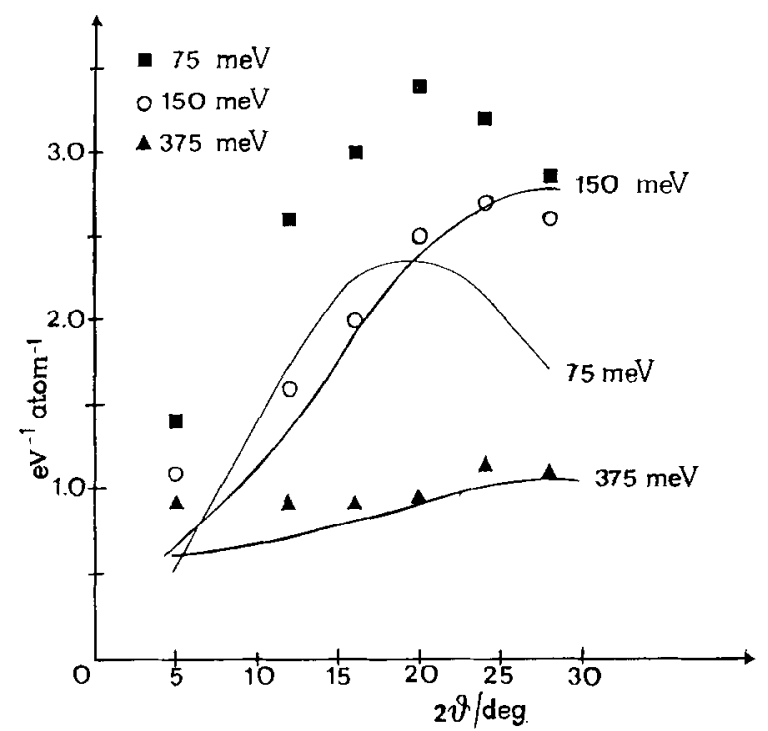

Fig. 5. The experimentally measured $S_{\text {exp }}^{\text {single }}(Q, \omega)$ compared with the results of the harmonic calculations, as a function of the scattering angle and for selected values of the energy $h \omega$; the solid line represents the result of the harmonic calculations.

is strong disagreement between experiment and theory at the librational frequency. Both these latter findings are confirmed by the $\mathrm{KH}_{2} \mathrm{PO}_{4}$ experiment [4]. In this sample one has an ordered phase below $120 \mathrm{~K}$ and a disordered one above. The $g(\omega)$ function is shown in Fig. 6 at $T=70 \mathrm{~K}$ and $T=150 \mathrm{~K}$. From 


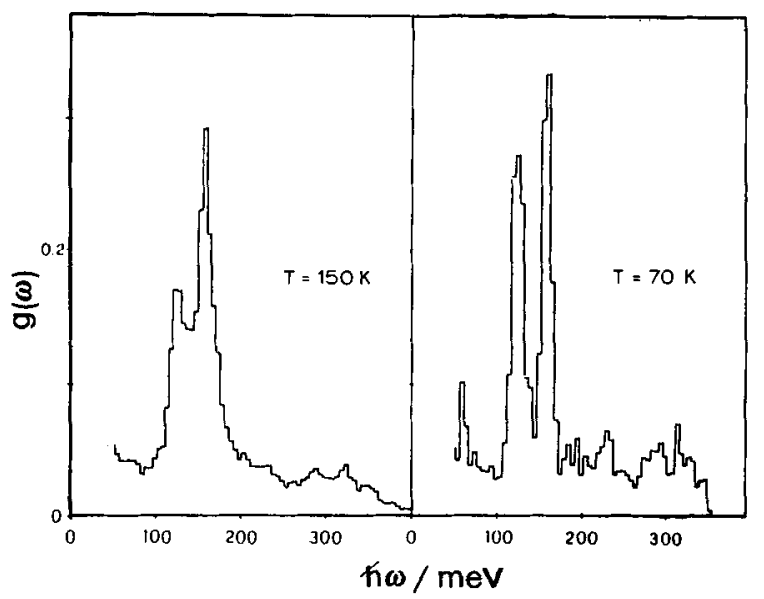

Fig. 6. The hydrogen projected density of states, $g(\omega)$, for polycrystalline $\mathrm{KH}_{2} \mathrm{PO}_{4}$ (a) at $T=150$ $\mathrm{K}$ and (b) at $T=70 \mathrm{~K}$, in the intramolecular vibrational region [4].

this figure we note that in the low temperature measurement more structures are resolved in the bending $(E=150 \mathrm{meV})$ and stretching $(E=300 \mathrm{meV})$ bands. However, the overall band widths remain the same. The anharmonic behavior of the proton motion cannot account for the features observed since the temperature difference between the two measurements is too small; indeed a shorter run at $36 \mathrm{~K}$ has shown very little change from the measurements at $T=70 \mathrm{~K}$. A conclusion which can then be drawn from this result is that the force constants are dependent on the local configuration of the hydrogen bonds; in this way the various configurations present in the disordered phase produce fluctuations of the mode frequency, thus smearing the structure resolved in the ordered phase.

As a final example of experiments of this kind, a study of the proton dynamics in polycrystalline $\mathrm{D}_{2} \mathrm{O}$ is presented. In this case one can derive the density of states, only in the stretching region, where the incoherent approximation holds [3], and see a possible influence of the mass variation on the proton dynamics. In Figs. $7(\mathrm{a})-7(\mathrm{~b})$, the $g(\omega)$ (open circles) and $\omega(q)$ for the O-D stretching band are plotted for $\mathrm{D}_{2} \mathrm{O}[3,11]$. The overall width of this band is about $42 \mathrm{meV}$ and its shape is as featureless as in the case of polycrystalline ice $I_{h}$ (full circles), with all bands shifted in energy by a factor $\sqrt{ } 2$, owing to the mass difference between deuterons and protons. Furthermore the $\mathrm{O}-\mathrm{D}$ stretching band spreads over the same $\omega$-range as the coherent experiment (Fig. 7(b)). In this case a check according to eqns. (4a) and (4b), as for $\mathrm{H}_{2} \mathrm{SO}_{4}$, has proved that anharmonicity is not responsible for the observed band width.

Therefore one can conclude that neither the disorder nor anharmonicity are the leading mechanisms responsible for the breadth of the intramolecular density of states in ice and ice-like solids. The appreciable breadth of the vibra- 

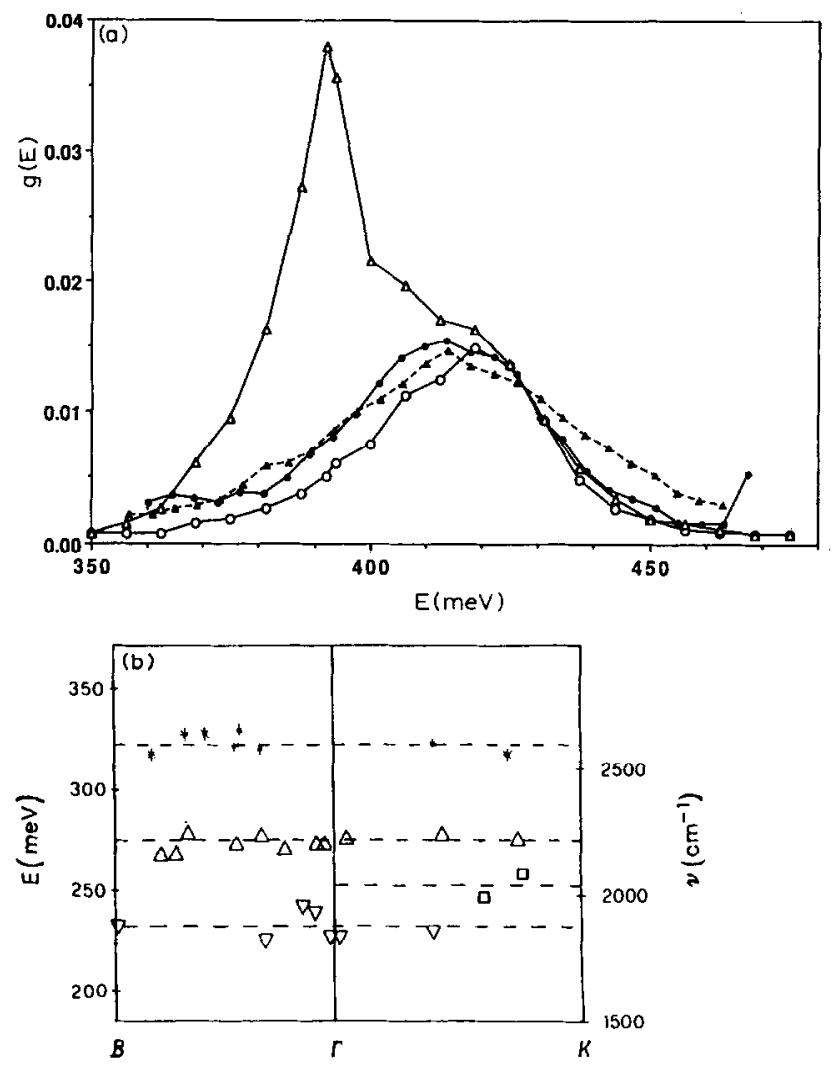

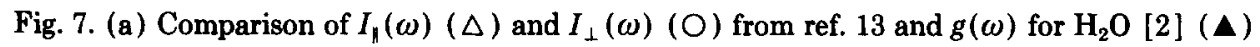
and $\mathrm{D}_{2} \mathrm{O}$ [3] (O). (b) Phonon dispersion curves for a deuterated single crystal of ice $\mathrm{I}_{h}$ in the stretching region [11].

tional $g(\omega)$ bands in these systems can be ascribed to the presence of $\mathrm{H}$-bonds $[4,5,12]$. The disorder of the hydrogen-bond network can eventually produce some fluctuation of the force constants, which can affect the vibrational spectrum, smearing out its structures. The anharmonic coupling of the bending and stretching with the translational phonons, which could be operative at some of the temperatures considered, are shown to give very little contribution.

A further comment can be made comparing the neutron and light scattering experiments, in the case of ice. In Fig. $7(a)$ the $g(\omega)$ of light and heavy ice in the stretching region, are presented together with the polarized $I_{\| \mid}(\omega)$, and depolarized $I_{\perp}(\omega)$ Raman spectra of ice [13]. The intensity of the neutron scattering data have been scaled to take into account the difference in mass and frequency of the stretching band occurring for $\mathrm{O}-\mathrm{D}$ and $\mathrm{O}-\mathrm{H}$. The light scattering data have been multiplied by an arbitrary intensity factor. There is good agreement between the depolarized Raman and neutron spectral shapes, 
both giving a stretching band width of $40-45 \mathrm{meV}$. On the contrary the polarized Raman spectrum displays a strong intensity at a frequency of about 390 $\mathrm{meV}$. These findings suggest that the depolarized Raman spectrum can be closely related to the vibrational density of states which extends from 350 to $450 \mathrm{meV}$. The strongly polarized feature at about $390 \mathrm{meV}$ is suggested to show up in the $I_{\|}(\omega)$ as the result of a large net polarizability change due to a collective in-phase stretching of the water molecule [13].

\section{REFERENCES}

1 G.H. Lander and D.L. Price, Physics Today, 38 (1985) 38.

2 C. Andreani, P. Bosi, F. Sacchetti and C.K. Loong, J. Chem. Phys., 83 (1985) 750.

3 C. Andreani, J. Mayers, P. Postorino and M.A. Ricci, Mol. Phys., 73 (1991) 737.

4 C. Andreani, P. Bisanti, C. Petrillo, F. Sacchetti, B.C. Boland, Z.A. Bowden and A.D. Taylor, Mol. Phys., 68 (1989) 681.

5 C. Andreani, V. Merlo, M.A. Ricci and B.C. Boland, Mol. Phys., 66 (1989) 747.

6 S. Lovesey, Theory of Neutron Scattering from Condensed Matter, Vol. 1, Int. Ser. Monographs on Physics 72, Clarendon Press, Oxford, 1984, p. 120.

7 C. Andreani, V. Merlo, M.A. Ricci and D. Lepoire, Nucl. Instrum. Methods, in press.

8 C. Andreani, V. Merlo and M.A. Ricci, Nucl. Instrum. Methods, Ser. B, 36 (1989) 216.

9 J.R.D. Copley, D.L. Price and J.M. Rowe, Nucl. Instrum. Methods, 107 (1973) 501.

10 C.G. Windsor, Pulsed Neutron Scattering, Taylor and Francis, London, 1985.

11 C. Andreani, P. Bosi, E. Mazzega, F. Sacchetti and C.G. Windsor, J. Phys. C, 16 (1983) 3055.

12 T.C. Sivakumar, S.A. Rice and M. Sceats, J. Chem. Phys., 68 (1978) 3468.

13 L. Green, A.R. Lacey and M.G. Sceats, J. Chem. Phys., 90 (1986) 3958. 